\title{
Síndrome de ortodeoxia-platipnea y embolia luego de endocarditis derecha complicada: consecuencias del Foramen Oval Permeable.
}

\author{
Anibal Arias, Diego Funes, Pablo Oberti, Rodolfo Pizarro, Mariano Falconi, César Belziti
}

Instituto de Medicina Cardiovascular, Cardiología. Hospital Italiano

de Buenos Aires, Argentina.

\section{Introducción:}

Se presenta el caso clínico de una mujer de 63 años de edad con Endocarditis de la Válvula Tricúspide que desarrolló tromboembolismo pulmonar y un síndrome de platipnea-ortodeoxia, explicado por la presencia de un formen oval permeable. La paciente se recuperó satis- factoriamente después de un reemplazo de la válvula tricúspide.

\section{Palabras Clave:}

Endocarditis Infecciosa - embolia paradójica- síndrome de platipnea-ortodeoxia. 


\section{Platypnea-Orthodeoxia syndrome and embolism after complicated right side endocarditis}

The case of 63 year old woman with Endocarditis at the Tricuspid Valve, pulmonary and paradoxical embolism with platypnea-orthodeoxia syndrome is presented. The patient recovered after surgical repla- cement of the tricuspid valve.

Keywords: Infective endocarditis, Paradoxical embolization, platypnea-orthodeoxia syndrome

\section{Introducción}

Una mujer de 63 años fue internada en nuestra institución por un cuadro de fiebre, astenia y pérdida de peso en el último mes. No refería antecedentes de enfermedad cardiovascular. Al examen físico se destacaba taquicardia sinusal y un soplo sistólico en foco tricuspídeo. El laboratorio mostraba 29000 glóbulos blancos (90 \% de células polimorfonucleares), eritrosedimentación de $60 \mathrm{~mm} /$ hora y hemocultivos positivos para Streptococcus agalactiae. El ecocardiograma transtorácico (ETT) evidenciaba una gran masa móvil asociada a la válvula tricúspide con insuficiencia severa (figura 1). La paciente negaba adicción a drogas endovenosas. Con diagnóstico de Endocarditis Infecciosa (EI) comenzó tratamiento antibiótico con Ampicillina y Gentamicina de acuerdo a la sensibilidad del germen.

A pesar de tratamiento antibiótico efectivo, la paciente evolucionó con tos y fiebre persistente. Los cultivos de control fueron negativos. Se realizó una Tomografía de Tórax con contraste endovenoso que reveló embolia pulmonar (figura 2). Al mismo tiempo la paciente evolucionó con disnea que empeoraba al sentarse (platipnea) y desaturación arterial (ortodeoxia). Al día siguiente presentó visión borrosa en forma aguda y se diagnosticó una endoftalmitis. Se efectuó un ecocardiograma transesofágico (ETE) que evidenció la presencia de un foramen oval permeable (FOP) con aneurisma del tabique y pasaje de burbujas de derecha a izquierda (figura 3). Este estudio, confirmó además la presencia de una gran vegetación móvil asociada a la válvula tricúspide con insuficiencia de grado severo. Dado el cuadro de insuficiencia tricuspidea severa complicada con síndrome de ortodeoxia-platipnea, embolia pulmonar y paradojal, se realizó reemplazo de válvula tricuspidea y cierre del FOP. La paciente evolucionó favorablemente y se otorgó el alta hospitalaria. 
Síndrome de ortodeoxia-platipnea y embolia luego de endocarditis derecha complicada: consecuencias del Foramen Arias A., et al.

Figura 1. EI ETT muestra una gran masa móvil asociada a la válvula tricúspide (flechas).

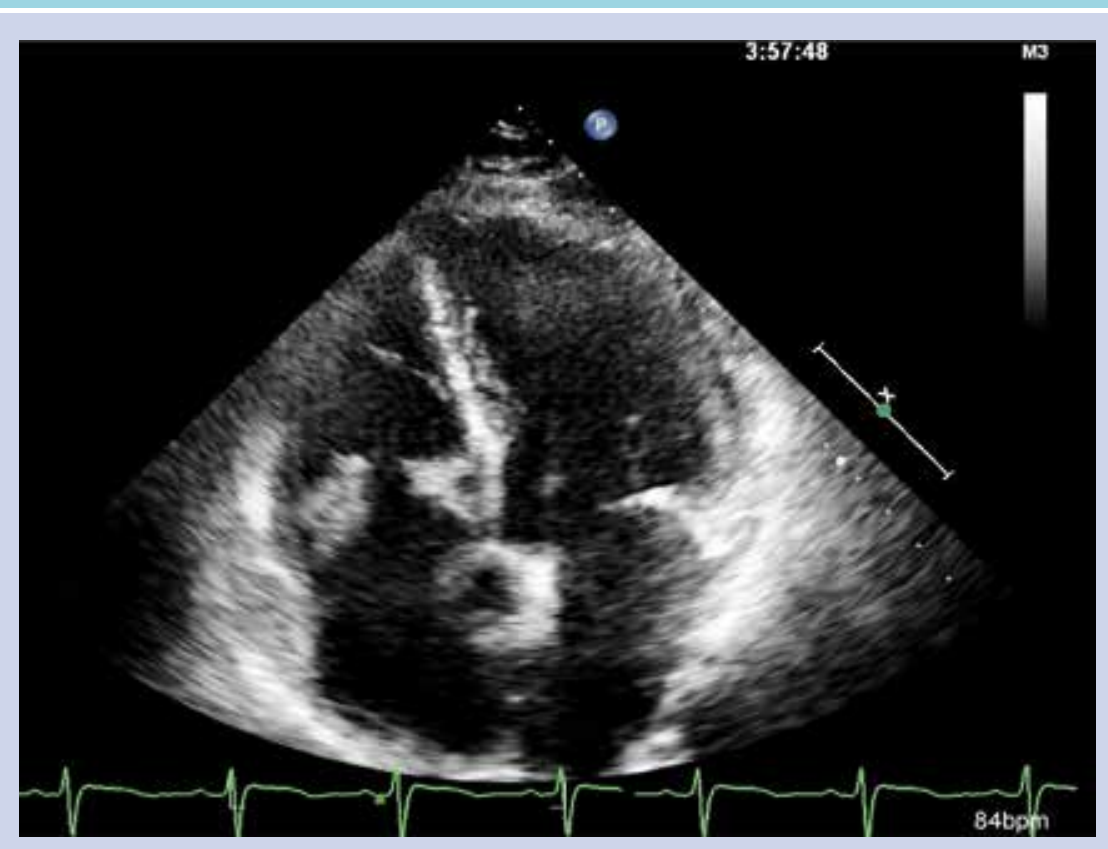

Figura 2. Tomografía de Tórax con contraste endovenoso que revela la presencia de embolia pulmonar (flecha)

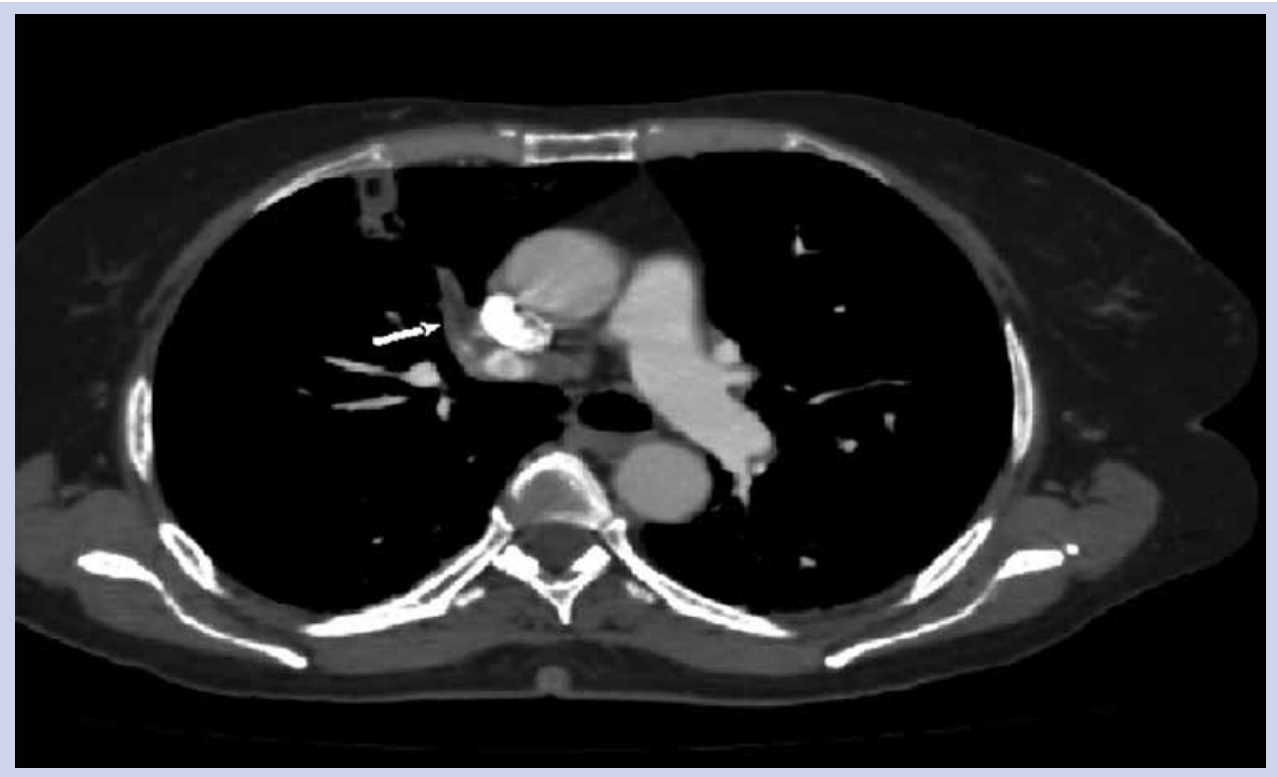




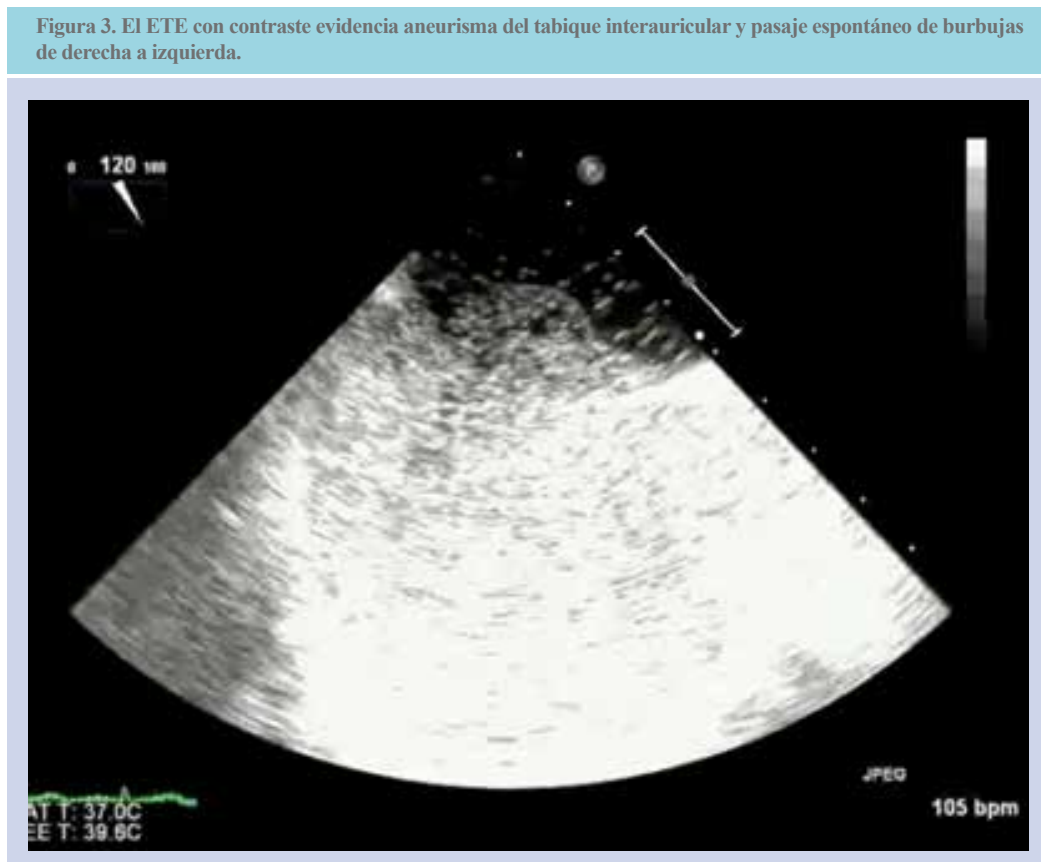

\section{Discusión}

La EI derecha es infrecuente en ausencia de cardiopatías congénitas, adicción a drogas endovenosas o catéteres en cavidades derechas.1 Además, en ocasiones la forma de presentación se asemeja a la de una infección respiratoria con lo cual el diagnóstico puede resultar un verdadero desafío.1 El germen aislado con mayor frecuencia es el Stafilococo Aureus y el Streptococo es más infrecuente. 1,2

En este caso en particular, no se pudo determinar el origen del foco infeccioso ni se pudo identificar ningún factor predisponente. Con respecto al tratamiento, en primera instancia se decidió por tratamiento médico conservador, pero luego dado la evolución complicada se optó por la resolución quirúrgica. En este sentido, en primer lugar, la paciente presentó embolia pulmonar séptica y paradojal en el territorio de la arteria oftálmica como consecuencia del FOP. Por otra parte y también como consecuencia del FOP, desarrolló un síndrome de ortodeoxia- platipnea confirmado por el pasaje de burbujas de derecha a izquierda en el ETE. Este síndrome es muy infrecuente y se ha asociado a diversas condiciones cardíacas y extracardiacas. 3 Turek y col. reportaron un caso de EI complicada con síndrome de ortodeoxia- platipnea que fue operado por este motivo.4 En este caso además de la complicación mencionada, se agrega la embolia pulmonar y paradójica.

\section{Referencias:}

1. REVILLA A, LÓPEZ J, VILLACORTA E, GÓMEZ I, SEVILLA T, DEL POZO MA, et al. Endocarditis derecha aislada en pacientes no adictos a drogas por vía parenteral. Rev Esp Cardiol. 2008; 61: 1253-9.

2. IWAMA T, SHIGEMATSU S, ASAMI K, KUBO I, KITAZUME H, TANABE S, et al. Tricuspid Valve Endocarditis with Large Vegetations in a Non-Drug Addict without Underlying
Cardiac Disease. Internal Medicine. 1996; 35: 203-206.

3. CHENG TO. Mechanisms of Platypnea-Orthodeoxia: What Causes Water to Flow Uphill?. Circulation 2002; 105: e47.

4. TUREK M, KAROVITHC A, AARON S, BRAIS M. Persistent hypoxemia occurring as a complication of tricuspid valve endocarditis. J Am Soc Echocardiogr 2000; 13: $412-4$ 